\title{
The scalar B meson in the static limit of HQET
}

\section{Benoît Blossier}

Laboratoire de Physique Théorique (Bât. 210), Université Paris Sud, Centre d'Orsay, 91405

Orsay-Cedex, France

\section{Nicolas Garon}

Department of Applied Mathematics \& Theoretical Physics, University of Cambridge, Wilberforce Road, Cambridge CB3 OWA, United Kingdom

\section{Antoine Gérardin*}

Laboratoire de Physique Théorique (Bât. 210), Université Paris Sud, Centre d'Orsay, 91405 Orsay-Cedex, France

E-mail: antoine.gerardineclermont.in2p3.fr

We present two-flavor lattice QCD estimates of the hadronic couplings $g_{B_{0}^{*} B \pi}$ and $g_{B_{1}^{*} B_{0}^{*} \pi}$ that parametrise the non leptonic decays $B_{0}^{*} \rightarrow B \pi$ and $B_{1}^{*} \rightarrow B_{0}^{*} \pi$. Our framework is the Heavy Quark Effective Theory (HQET) in the static limit and solving a Generalized Eigenvalue Problem (GEVP) reveals crucial to disentangle the $B_{0}^{*}\left(B_{1}^{*}\right)$ state from the $B \pi\left(B^{*} \pi\right)$ state. This work brings us some experience on how to treat the possible contribution from multihadronic states to correlation functions calculated on the lattice, especially when $S$-wave states are involved.

The 32nd International Symposium on Lattice Field Theory,

23-28 June, 2014

Columbia University New York, NY

\footnotetext{
* Speaker.
} 


\section{Introduction}

Heavy Meson Chiral Perturbation Theory (HM $\chi \mathrm{PT})[1,2]$ is commonly used to extrapolate lattice data in the heavy-light sector to the physical point. Relying on Heavy Quark Symmetry and the (spontaneously broken) chiral symmetry, an effective Lagrangian is derived where heavy-light mesons fields [3] couple to a Goldstone field via derivative operators. In the static limit, the total angular momentum of the light degrees of freedom, $\vec{j}_{l}=\vec{s}_{l}+\vec{L}$, is conserved independently of the total angular momentum $J=j_{l} \pm 1 / 2$. The pseudoscalar $(B)$ and the vector $\left(B^{*}\right)$ mesons belong to the doublet $j_{l}^{P}=(1 / 2)^{-}$corresponding to $L=0$ whereas the scalar $\left(B_{0}^{*}\right)$ and the axial $\left(B_{1}^{*}\right)$ mesons belong to the positive parity doublet $j_{l}^{P}=(1 / 2)^{+}$corresponding to $L=1$. At leading order, the effective Lagrangian is parametrized by three couplings $\hat{g}, \tilde{g}$ and $h$. The first coupling, $\hat{g}$, relates transitions between mesons belonging to the same doublet $J^{P}=(1 / 2)^{-}$and has been precisely measured on the lattice $[4,8]$. On the contrary, the last two couplings are less precisely known and there is only one direct lattice computation of $h$ and $\tilde{g}$ [9].

\section{Extraction of $\left\langle B \pi \mid B_{0}^{*}\right\rangle$}

The transition amplitude under interest is parameterized by

$$
\Gamma\left(B_{0}^{* 0} \rightarrow B^{+} \pi^{-}\right)=\frac{1}{8 \pi} g_{B_{0}^{*} B \pi}^{2} \frac{\left|\vec{q}_{\pi}\right|}{m_{B_{0}^{*}}^{2}}, \quad\left|\vec{q}_{\pi}\right|=\frac{\sqrt{\left[m_{B_{0}^{*}}^{2}-\left(m_{B}+m_{\pi}\right)^{2}\right]\left[m_{B_{0}^{*}}^{2}-\left(m_{B}-m_{\pi}\right)^{2}\right]}}{2 m_{B_{0}^{*}}},
$$

where $f_{\pi}$ is the pion decay constant. In particular, in the static limit, $g_{B_{0}^{*} B \pi}$ is related to the HM $\chi \mathrm{PT}$ coupling $h[10]$ via

$$
g_{B_{0}^{*} B \pi}=\left\langle\pi^{+}\left(q_{\pi}\right) B^{-}(p) \mid B_{0}^{* 0}\left(p^{\prime}\right)\right\rangle=\sqrt{\frac{m_{B}}{m_{B_{0}^{*}}}}\left(m_{B_{0}^{*}}^{2}-m_{B}^{2}\right) \frac{h}{f_{\pi}} .
$$

On the lattice, following the approach proposed in Ref [11], the Fermi golden rule reads

$$
\Gamma\left(B_{0}^{*} \rightarrow B^{-} \pi^{+}\right)=2 \pi\left|\left\langle\pi^{+}\left(q_{\pi}\right) B^{-}(p) \mid B_{0}^{* 0}\left(p^{\prime}\right)\right\rangle\right|^{2} \rho,
$$

where the density of states $\rho$, for a given energy $E_{\pi}$ and a lattice volume $L^{3}$, is $\rho\left(E_{\pi}\right)=\frac{L^{3}}{2 \pi^{2}}\left|\vec{q}_{\pi}\right| E_{\pi}$. Considering the matrix of two-point correlation functions $C_{B_{0}^{*} B \pi}^{(2)}(t)=\left\langle\mathscr{O}_{i}^{B \pi}(t) \mathscr{O}_{j}^{B_{0}^{* \dagger}}(0)\right\rangle$, where $O_{i}^{B_{0}^{*}}$ and $O_{j}^{B \pi}$ are interpolating fields with vanishing momenta of the $B_{0}^{*}$ and the $B \pi$ states, one has

$$
C_{B_{0}^{*} B \pi, i j}^{(2)}(t)=\sum_{t_{1}}\left\langle 0\left|\mathscr{O}_{i}^{B_{0}^{*}}\right| B_{0}^{*}\right\rangle x\left\langle B \pi\left|\mathscr{O}_{j}^{B \pi}\right| 0\right\rangle e^{-m_{B_{0}^{*} t_{1}}} e^{-m_{B \pi}\left(t-t_{1}\right)}+\mathscr{O}\left(x^{3}\right)+\text { excited states, }
$$

with $x=\left|a\left\langle\pi^{+}\left(q_{\pi}\right) B^{-}(p) \mid B_{0}^{* 0}\left(p^{\prime}\right)\right\rangle\right|$. We have assumed small overlaps $\left\langle 0\left|\mathscr{O}_{i}^{B_{0}^{*}}\right| B \pi\right\rangle$ and $\left\langle 0\left|\mathscr{O}_{j}^{B \pi}\right| B_{0}^{*}\right\rangle$ and the normalization of states is $\langle n \mid m\rangle=1$. Finally, close to the threshold, one can use the Generalized Eigenvalues Problem to extract $x$ from the ratio $[12,15]$

$$
R^{\mathrm{GEVP}}(t)=\frac{\left(v_{B_{0}^{*}}\left(t, t_{0}\right), C_{B_{0}^{*} B \pi}^{(2)}(t) v_{B \pi}\left(t, t_{0}\right)\right)}{\sqrt{\left(v_{B_{0}^{*}}\left(t, t_{0}\right), C_{B_{0}^{*} B_{0}^{*}}^{(2)}(t) v_{B_{0}^{*}}\left(t, t_{0}\right)\right) \times\left(v_{B \pi}\left(t, t_{0}\right), C_{B \pi B \pi}^{(2)}(t) v_{B \pi}\left(t, t_{0}\right)\right)}},
$$

where $C_{B_{0}^{*} B_{0}^{*}}^{(2)}$ and $C_{B \pi B \pi}^{(2)}$ are, respectively, matrices of two-point correlation functions of a scalar $B$ meson and a $B \pi$ multihadronic state and $v_{X}$ are the generalized eigenvectors associated to the ground state in the corresponding channel. 


\section{Extraction of $\tilde{g}$}

The coupling $\tilde{g}$ parametrises the transition between the scalar $B_{0}^{*}$ and the axial $B_{1}^{*}$ mesons by exchanging a single soft pion [10]

$$
\left\langle B_{0}^{*}(\vec{p}=\overrightarrow{0})\left|\bar{\psi}_{l} \gamma_{k} \gamma_{5} \psi_{l}\right| B_{1}^{*}\left(\vec{p}=\overrightarrow{0}, \varepsilon_{k}\right)\right\rangle=\tilde{g} \varepsilon_{k},
$$

where $\varepsilon_{k}$ is the polarization vector of the axial $B$ meson. This matrix element can be extracted using the same technique as discussed in [14] but applied to the first excited heavy-light mesons doublet. Therefore, following the method of $[14,15]$, we consider the ratio

$$
\mathscr{M}^{\mathrm{SGEVP}}\left(t, t_{0}\right)=-\partial_{t}\left(\frac{\left(v_{B_{0}^{*}}\left(t, t_{0}\right),\left[K(t) / \lambda_{B_{0}^{*}}\left(t, t_{0}\right)-K\left(t_{0}\right)\right] v_{B_{1}^{*}}\left(t, t_{0}\right)\right)}{\left(v_{B_{0}^{*}}\left(t, t_{0}\right), C_{B_{0}^{*} B_{0}^{*}}^{(2)}\left(t_{0}\right) v_{B_{0}^{*}}\left(t, t_{0}\right)\right)^{1 / 2}\left(v_{B_{1}^{*}}\left(t, t_{0}\right), C_{B_{1}^{*} B_{1}^{*}}^{(2)}\left(t_{0}\right) v_{B_{1}^{*}}\left(t, t_{0}\right)\right)^{1 / 2}}\right),
$$

where $K_{i j}(t)$ is the summed three-point correlation function

$$
K_{i j}(t)=\sum_{t_{1}} C_{i j}^{(3)}\left(t, t_{1}\right), \quad C_{i j}^{(3)}\left(t, t_{1}\right)=\frac{1}{V^{3}} \sum_{\vec{x}, \vec{y}, \vec{z} t_{x}} \sum_{\mathcal{O}_{i}}\left\langle\mathscr{O}_{i}^{B_{0}^{*}}\left(\vec{z}, t+t_{x}\right) \mathscr{A}_{k}\left(\vec{y}, t_{1}+t_{x}\right) \mathscr{O}_{j}^{B_{1}^{*} \dagger}\left(\vec{x}, t_{x}\right)\right\rangle,
$$

and $\mathscr{A}_{k}=Z_{A} \bar{\psi}_{l}(x) \gamma_{k} \gamma_{5} \psi_{l}(x)$ is the renormalized axial current. The renormalisation constant $Z_{A}$ was determined non-perturbatively by the ALPHA Collaboration [16]. Finally, one can show that [14]

$$
\mathscr{M}^{\mathrm{sGEVP}}\left(t, t_{0}\right) \underset{t_{0}=t-1}{\stackrel{t \gg 1}{\longrightarrow}} \tilde{g}+\mathscr{O}\left(t e^{-\Delta_{N+1, n} t}\right)
$$

where $\Delta_{m n}=E_{m}-E_{n}$ is the energy difference between the $m^{\text {th }}$ and $n^{\text {th }}$ excited states of the GEVP and $N \times N$ is the size of the matrix of correlators defining the GEVP.

\section{Lattice setup}

In our study we have performed measurements on a subset of four $\mathrm{N}_{\mathrm{f}}=2$ CLS lattice simulations satisfying the criteria $m_{B_{0}^{*}} \approx m_{B \pi}$. The main parameters are collected in Table 1 . They use the plaquette gauge action, the non perturbatively $\mathscr{O}(a)$ improved Wilson-Clover fermions for the light quark and the HYP2 discretisation for the static quarks [17]. Static-light correlation functions are computed using stochastic all-to-all propagators with full time dilution [18] and we have implemented both static-light meson and multihadronic state interpolating fields, respectively given by

$$
\begin{aligned}
& O_{\Gamma, n}^{B}=\bar{\psi}_{l}^{(n)} \Gamma \psi_{h}, \quad \Gamma=\left\{\gamma_{0}, \gamma_{5}, \gamma_{0} \sum_{i=1}^{3} \gamma_{i} \nabla_{i}, \gamma_{5} \sum_{i=1}^{3} \gamma_{i} \nabla_{i}\right\} \\
& \mathscr{O}_{n}^{B \pi}=\frac{1}{V^{2}} \sum_{\vec{x}_{1}, \vec{x}_{2}} \sqrt{\frac{2}{3}}\left[\bar{\psi}_{d} \gamma_{5} \psi_{u}\right]\left(x_{1}\right)\left[\bar{\psi}_{u}^{(n)} \gamma_{5} \psi_{h}\right]\left(x_{2}\right)-\sqrt{\frac{1}{6}}\left[\bar{\psi}_{u} \gamma_{5} \psi_{u}-\bar{\psi}_{d} \gamma_{5} \psi_{d}\right]\left(x_{1}\right)\left[\bar{\psi}_{d}^{(n)} \gamma_{5} \psi_{h}\right]\left(x_{2}\right) .
\end{aligned}
$$

Here, $\psi_{h}$ is the static quark field and $\psi_{l}^{(n)} \equiv\left(1+\kappa_{G} a^{2} \Delta\right)^{R_{n}} \psi_{l}$ is the relativistic quark field $(l=u / d)$ with $R_{n}$ steps of Gaussian smearing [19] ( $\left.\kappa_{G}=0.1, r_{n} \equiv 2 a \sqrt{\kappa_{G} R_{n}} \leq 0.6 \mathrm{fm}\right)$. We have computed the following two-point correlation functions on the lattice:

$$
C_{B_{0}^{*} B_{0}^{*}}^{n m}(t), \quad C_{B \pi B \pi}^{n m}(t), \quad C_{B \pi B_{0}^{*}}^{n m}(t), \quad C_{B_{0}^{*} B \pi}^{n m}(t),
$$




\begin{tabular}{|c|c|c|c|c|c|c|c|}
\hline CLS label & $\beta$ & $L^{3} \times T$ & $\kappa$ & $a[\mathrm{fm}]$ & $m_{\pi}[\mathrm{MeV}]$ & $\#_{h}$ & $\#_{\tilde{g}}$ \\
\hline B6 & 5.2 & $48^{3} \times 96$ & 0.13597 & 0.075 & 280 & 250 & 200 \\
\hline E5 & 5.3 & $32^{3} \times 64$ & 0.13625 & 0.065 & 435 & 450 & 400 \\
F6 & & $48^{3} \times 96$ & 0.13635 & & 310 & 300 & 250 \\
\hline N6 & 5.5 & $48^{3} \times 96$ & 0.13667 & 0.048 & 340 & 250 & 200 \\
\hline
\end{tabular}

Table 1: Simulations parameters: the bare coupling constant $\beta=6 / g_{0}^{2}$, spatial extent in lattice units $L$ (with $T=2 L$ ), hopping parameter $\kappa$, lattice spacing $a$, pion mass $m_{\pi}$ and number of configurations used for the computation of the two-point and three-point correlation functions respectively.
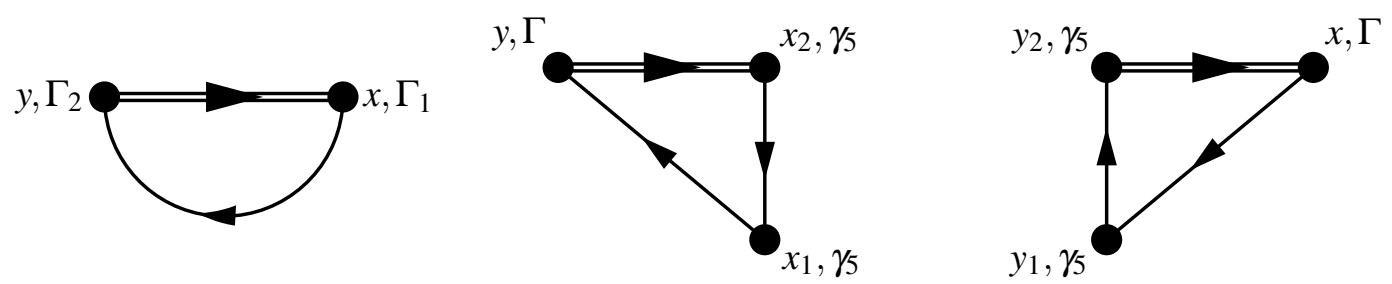

Figure 1: (Left) Diagram representing the correlator $C_{B_{0}^{*} B_{0}^{*}}^{n m}$. (Right) Diagrams contributing to the correlators $C_{B \pi B_{0}^{*}}^{n m}$ and $C_{B_{0}^{*} B \pi}^{n m}$. Simple and double lines represent the light and static quark propagators respectively.
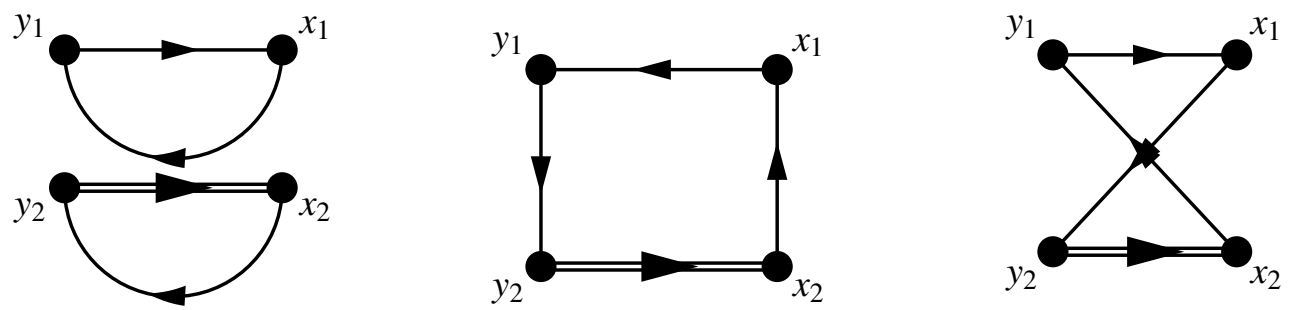

Figure 2: Direct, box and cross diagrams contributing to the correlator $C_{B \pi B \pi}^{n m}$.

where $n, m$ corresponds to the level of smearing. The diagrams entering the computation are depicted in Figures 1 and 2. Since the box and cross diagrams require at least one more inversion of the Dirac operator for each time slice, they have been computed only in the case of the ensemble E5 and their contribution (of the order of one percent) appears to be negligible at our level of precision. Finally, we have also computed the three point correlation functions (3.2) needed for the extraction of the coupling $\tilde{g}$ using the same basis of interpolating operators.

\section{Results}

The results for $h$ and $g_{B_{0}^{*} B \pi}$ are collected in Table 2 where we used the value $m_{B_{0}^{*}}-m_{B}=$ $385(17)_{\text {stat }}(28)_{\text {syst }} \mathrm{MeV}$ [20] that we extract on the same ensembles. Concerning the three-point correlation functions, we observed that the signal is less noisy for interpolating operators built from the insertion of a covariant derivative as shown in Figure 3. Therefore, only those fields are used in the final result. The light-quark mass and lattice spacing dependence is small, therefore we try a fit with a constant and we obtain $h=0.84(3)$ and $\tilde{g}=-0.120$ (3). Performing a linear fit in $m_{\pi}^{2}$, we get compatible results $h=0.86(4)$ and $\tilde{g}=-0.122(8)$. A third possibility is to use the 

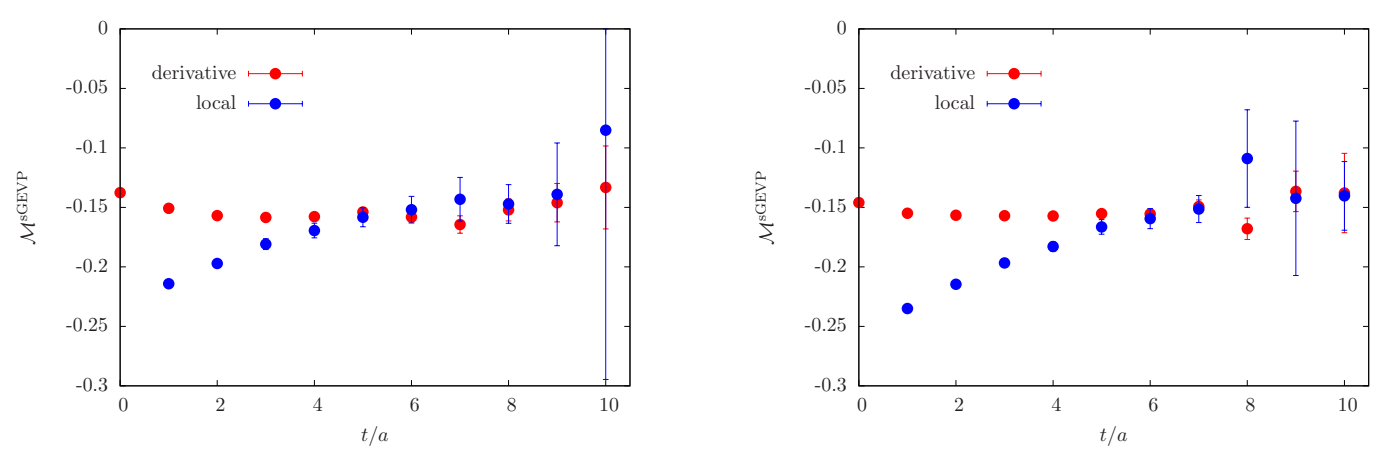

Figure 3: Comparison of the signal obtained for $\mathscr{M}^{\mathrm{SGEVP}}$ using local (blue) and derivative (red) interpolating operators for the ensembles E5 (left) and F6 (right).

\begin{tabular}{|c|c|c|c|c|c|c|}
\hline CLS & $a x$ & $\Gamma /\left|\vec{q}_{\pi}\right|$ & $g_{B_{0}^{*} B \pi}[\mathrm{GeV}]$ & $a \delta$ & $h$ & $\tilde{g}$ \\
\hline B6 & $-0.0156(4)$ & $0.92(4)$ & $27.4(0.1)(0.6)$ & $0.141(4)$ & $0.85(3)(2)$ & $-0.122(7)$ \\
E5 & $-0.0238(9)$ & $0.86(7)$ & $26.4(0.1)(1.0)$ & $0.133(6)$ & $0.82(3)(3)$ & $-0.117(6)$ \\
F6 & $-0.0161(3)$ & $0.95(3)$ & $27.7(0.1)(0.5)$ & $0.129(3)$ & $0.86(3)(2)$ & $-0.119(4)$ \\
N6 & $-0.0172(6)$ & $0.88(6)$ & $26.6(0.1)(0.9)$ & $0.092(3)$ & $0.82(3)(3)$ & $-0.122(5)$ \\
\hline
\end{tabular}

Table 2: Numerical values of $a x, \Gamma /\left|\vec{q}_{\pi}\right|, g_{B_{0}^{*} B \pi}, \delta=m_{B_{0}^{*}}-m_{B}, h$ and $\tilde{g}$ obtained on the four CLS ensembles that we have analyzed, with $m_{B_{0}^{*}} \approx m_{B}+m_{\pi}$.

NLO formulae of HM $\chi$ PT [21]. However, for our lattice ensembles, the pion mass lies in the range [280-440] MeV and the mass difference between the scalar $B$ meson and the ground state $B$ meson is of the order of $\delta \sim 400 \mathrm{MeV}$. Therefore, the heavy-light states of opposite parity should be taken into account [9]. Their contributions have been computed in [22] and we tried the fit formulae

$$
\begin{aligned}
& h=h_{0}\left[1-\frac{3}{4} \frac{3 \hat{g}_{0}^{2}+3 \tilde{g}_{0}^{2}+2 \hat{g}_{0} \tilde{g}_{0}}{\left(4 \pi f_{\pi}\right)^{2}} m_{\pi}^{2} \log \left(m_{\pi}^{2}\right)-\frac{h_{0}^{2}}{\left(4 \pi f_{\pi}\right)^{2}} \frac{m_{\pi}^{2}}{2 \delta^{2}} m_{\pi}^{2} \log \left(m_{\pi}^{2}\right)\right]+C_{h} m_{\pi}^{2}, \\
& \tilde{g}=\tilde{g}_{0}\left[1-\frac{2+4 \tilde{g}_{0}^{2}}{\left(4 \pi f_{\pi}\right)^{2}} m_{\pi}^{2} \log \left(m_{\pi}^{2}\right)+\frac{h_{0}^{2}}{\left(4 \pi f_{\pi}\right)^{2}} \frac{m_{\pi}^{2}}{8 \delta^{2}}\left(3+\frac{\hat{g}_{0}}{\tilde{g}_{0}}\right) m_{\pi}^{2} \log \left(m_{\pi}^{2}\right)\right]+C_{\tilde{g}} m_{\pi}^{2},
\end{aligned}
$$

where $\hat{g}_{0}=0.5(1)[4,8]$ is the pionic couplings associated to $H^{*} \rightarrow H \pi$ and the mass difference $\delta$ is given in Table 2. The results are $h=0.84(3)$ and $\tilde{g}=-0.116(7)$ and we show in Figure (4) the chiral extrapolations of $h$ and $\tilde{g}$. The quark mass dependence is very small and the influence of the chiral logarithms does not change our result significantly, we quote finally

$$
h=0.84(3)(2), \quad \tilde{g}=-0.122(8)(6),
$$

In Refs. [12, 13], an alternative method to evaluate such a coupling like $h$ was proposed. Indeed, one can show that the connected contribution to the correlation function $C_{B \pi} B \pi(t)$, which includes box and cross diagrams, has the following behavior:

$$
\widetilde{R}(t)=\frac{\left(v_{B \pi}(t), C_{\text {connected }}(t) v_{B \pi}(t)\right)}{\left(v_{B \pi}(t), C_{B \pi B \pi}(t) v_{B \pi}(t)\right)}=A^{\prime}+\frac{1}{2} x^{2} t^{2}+\mathscr{O}(t),
$$

where $C_{\text {conected }}(t)=-\frac{3}{2} C_{\text {box }}(t)+\frac{1}{2} C_{\text {cross }}(t)$. These diagrams have been computed only for the CLS ensemble E5 and the result reads $|a x|=0.0237(8)$, in perfect agreement with the one obtained 

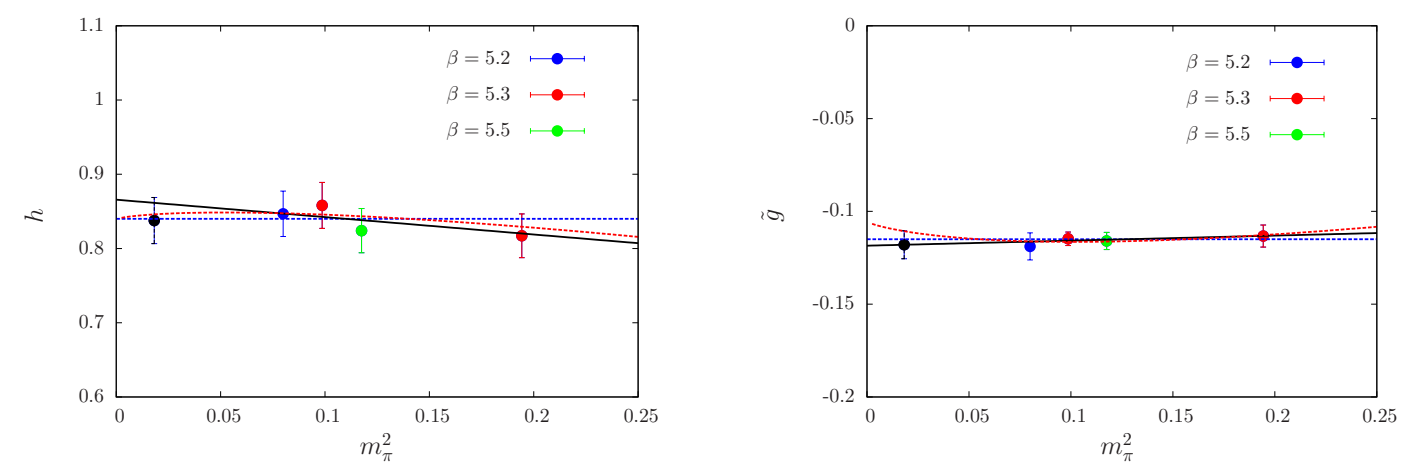

Figure 4: Chiral extrapolations of the effective couplings $h$ (left) and $\tilde{g}$ (right). The dashed blue line corresponds to the constant fit, the black line corresponds to the linear fit and the dashed red line corresponds to the fit formulae (5.1), (5.2) with the expression derived in $\mathrm{HM} \chi \mathrm{PT}$.

by the previous method (see Table 2). The fit range has been varied from $t / a \in[9-18]$ to $t / a \in$ $[13-18]$ where the result is stable to estimate the error.

\section{Discussion and conclusion}

The couplings $h$ and $\tilde{g}$ were explicitly computed on the lattice in Ref [9] and we confirm the small value of $\tilde{g}$. In particular this coupling for positive parity states is smaller than in the case of negative parity states $\tilde{g} \ll g$. For $h$, two results are reported for the two different actions used there: $h=0.69(2)\left({ }_{-7}^{+11}\right)$ and $h=0.58(2)\left({ }_{-2}^{+6}\right)$. These smaller results than ours might be explained by the larger quark masses simulated at that time: indeed the chiral extrapolation tends to lower the extrapolated value. Our result is also a bit larger than the QCD sum rules estimates: in Ref [23] the computation of $g_{B_{0}^{*} B \pi}$ gives $h=0.56(28)$, while in Ref [24] it gives $h=0.74(23)$.

We can also compare our finding with experimental data in the $D$ sector, although the static approximation of HQET is expected to give only a rough estimate due to quite large $1 / m_{c}$ corrections. With $m_{D_{0}^{*}}=2318(29) \mathrm{MeV}, \Gamma_{D_{0}^{*}}=267(40) \mathrm{MeV}$ [25] and assuming that the branching ratio $\mathscr{B}\left(D_{0}^{*} \rightarrow D \pi\right)$ is $\sim 100 \%$, we obtain $h=0.74(8)$, compatible with our result within error bars. In Ref. [26] the phase shift of the $D \pi$ scattering state was computed on the lattice: relating the coupling $g_{D_{0}^{*} D \pi}$ quoted in that paper to $h$, one finds that $h$ is around 1 .

In conclusion, we have extracted from lattice simulations with $\mathrm{N}_{\mathrm{f}}=2$ dynamical quarks the couplings $h$ and $\tilde{g}$ that parametrise the emission of a soft pion by a scalar $B$ meson and our results read $h=0.84(3)(2), \tilde{g}=-0.122(8)(6)$. The large value of $h$ compared to $\hat{g} \sim 0.5$ outlines the fact that some care is necessary to apply $\mathrm{HM} \chi \mathrm{PT}$ for pion masses close to mass splitting $m_{B_{0}^{*}}-m_{B} \sim 400 \mathrm{MeV}: B$ meson orbital excitation degrees of freedom cannot be neglected in chiral loops.

\section{Acknowledgements}

We thank Damir Becirevic and Chris Michael for valuable discussions and our colleagues in the CLS effort for the joint production and use of gauge configurations. Computations of the relevant correlation functions are made on GENCI/CINES, under the Grants 2013-056806 and 2014056806. 


\section{References}

[1] M. B. Wise, Phys. Rev. D 45, 2188 (1992).

[2] T. -M. Yan, H. -Y. Cheng, C. -Y. Cheung, G. -L. Lin, Y. C. Lin and H. -L. Yu, Phys. Rev. D 46, 1148 (1992), [Erratum-ibid. D 55, 5851 (1997)].

[3] A. F. Falk, H. Georgi, B. Grinstein and M. B. Wise, Nucl. Phys. B 343, 1 (1990).

[4] H. Ohki, H. Matsufuru, and T. Onogi, Phys. Rev. D 77, 094509 (2008). [arXiv:0802.1563 [hep-lat]].

[5] D. Becirevic, B. Blossier, E. Chang, and B. Haas, Phys. Lett. B 679, 231 (2009). [arXiv:0905.3355 [hep-lat]].

[6] W. Detmold, C. D. Lin, and S. Meinel, Phys. Rev. D 85, 114508 (2012). [arXiv:1203.3378 [hep-lat]].

[7] D. Becirevic and F. Sanfilippo, Phys. Lett. B 721, 94 (2013). [arXiv:1210.5410 [hep-lat]].

[8] F. Bernardoni, J. Bulava, M. Donnellan, and R. Sommer [ALPHA Collaboration], arXiv:1404.6951 [hep-lat].

[9] D. Becirevic, E. Chang and A. Le Yaouanc, [arXiv:1203.0167 [hep-lat]].

[10] R. Casalbuoni, A. Deandrea, N. Di Bartolomeo, R. Gatto, F. Feruglio and G. Nardulli, Phys. Rept. 281, 145 (1997). [hep-ph/9605342].

[11] C. McNeile et al. [UKQCD Collaboration], Phys. Rev. D 70, 054501 (2004). [hep-lat/0404010].

[12] C. McNeile et al. [UKQCD Collaboration], Phys. Rev. D 63, 114503 (2001). [hep-lat/0010019].

[13] C. McNeile et al. [UKQCD Collaboration], Phys. Lett. B 556, 177 (2003). [hep-lat/0212020].

[14] J. Bulava, M. Donnellan and R. Sommer, JHEP 1201, 140 (2012). [arXiv:1108.3774 [hep-lat]].

[15] B. Blossier, J. Bulava, M. Donnellan and A. Gérardin, Phys. Rev. D 87, no. 9, 094518 (2013). [arXiv:1304.3363 [hep-lat]].

[16] P. Fritzsch, F. Knechtli, B. Leder, M. Marinkovic, S. Schaefer, R. Sommer and F. Virotta, Nucl. Phys. B 865 (2012) 397 [arXiv:1205.5380 [hep-lat]].

[17] A. Hasenfratz and F. Knechtli, Phys. Rev. D 64, 034504 (2001). [hep-lat/0103029]; M. Della Morte, A. Shindler and R. Sommer, JHEP 0508, 051 (2005). [hep-lat/0506008].

[18] J. Foley, K. Jimmy Juge, A. O’Cais, M. Peardon, S. M. Ryan and J. -I. Skullerud, Comput. Phys. Commun. 172, 145 (2005). [hep-lat/0505023].

[19] S. Gusken, U. Low, K. H. Mutter, R. Sommer, A. Patel and K. Schilling, Phys. Lett. B 227, 266 (1989).

[20] D. Becirevic et al, in preparation.

[21] W. Detmold, C.-J. D. Lin and S. Meinel, Phys. Rev. D 84, 094502 (2011). [arXiv:1108.5594 [hep-lat]].

[22] S. Fajfer and J. F. Kamenik, Phys. Rev. D 74, 074023 (2006). [hep-ph/0606278].

[23] P. Colangelo, F. De Fazio, G. Nardulli, N. Di Bartolomeo and R. Gatto, Phys. Rev. D 52, 6422 (1995). [hep-ph/9506207].

[24] T. M. Aliev and M. Savci, J. Phys. G 22, 1759 (1996), [J. Phys. G 22, 1759 (1996)]. [hep-ph/9604258].

[25] J. Beringer et al. [Particle Data Group Collaboration], Phys. Rev. D 86, 010001 (2012).

[26] D. Mohler, S. Prelovsek and R. M. Woloshyn, Phys. Rev. D 87, no. 3, 034501 (2013). [arXiv:1208.4059 [hep-lat]]. 